\title{
Analysis of Autoantibodies Related to Tumor Progression in Sera from Patients with High-grade Non-muscle-invasive Bladder Cancer
}

\author{
SHO MINAMI ${ }^{1}$, KAZUMASA MATSUMOTO $^{2}$, RYO NAGASHIO $^{1,3}{ }^{,}$DAISUKE HAGIUDA ${ }^{1}$, ERIKO FUKUDA ${ }^{4}$, \\ NAOKI GOSHIMA ${ }^{4}$, MANABU HATTORI ${ }^{1,5}$, BENIO TSUCHIYA ${ }^{1,6}$, KAZUO HACHIMURA $^{3}$, \\ SHI-XU JIANG ${ }^{7}$, MAKOTO SAEGUSA ${ }^{7}$, MASATSUGU IWAMURA ${ }^{2}$ and YUICHI SATO ${ }^{1,3}$ \\ ${ }^{1}$ Department of Applied Tumor Pathology, Graduate School of Medical Sciences, \\ Kitasato University, Sagamihara, Japan; \\ Departments of ${ }^{3}$ Molecular Diagnosis, ${ }^{5}$ Clinical Cytology and ${ }^{6}$ Pathology, \\ School of Allied Health Sciences, Kitasato University, Sagamihara, Japan; \\ Departments of ${ }^{2}$ Urology and ${ }^{7}$ Pathology, School of Medicine, Kitasato University, Sagamihara, Japan; \\ ${ }^{4}$ Functional Proteomics Team, Molecular Profiling Research Center for Drug Discovery, \\ National Institute of Advanced Industrial Science and Technology, Tokyo, Japan
}

\begin{abstract}
Background/Aim: Bladder cancer (BC) has a high recurrence rate and may progress to being a muscle-invasive lesion, that is potentially associated with a poor prognosis. We identified tumor-associated proteins that were recognized by autoantibodies in sera from patients with high-grade nonmuscle-invasive bladder cancer (HG-NMIBC) by proteomic analysis. Materials and Methods: The serum levels of these autoantibodies against identified proteins were validated by dot blot analysis with sera from 95 patients with $B C$ and 35 healthy controls. The expression of identified proteins was immunohistochemically analyzed in 115 BC tissues. Results: Autoantibody against protein phosphatase 1, catalytic subunit, alpha isoform (PPPICA) protein was detected in pretreated sera from patients with $H G-N M I B C$ who showed progression. The serum IgG level of anti-PPPICA autoantibody was significantly correlated with pathological stage, grade, lymphovascular invasion, and prognosis. The immunoreactions for PPPICA protein in BC was significantly correlated with pathological stage, grade, and lymphovascular invasion. Conclusion: PPPICA is a candidate sero-diagnostic and prognostic marker for patients with $B C$.
\end{abstract}

Correspondence to: Kazumasa Matsumoto, MD, Department of Urology, Kitasato University School of Medicine,1-15-1 Kitasato, Minami-ku, Sagamihara, Kanagawa, 252-0374, Japan. Tel: +81 427789091, Fax: +81 427789374, e-mail: kazumasa@cd5.sonet.ne.jp

Key Words: Bladder cancer, serum, biomarker, autoantibody, PPP1CA.
The incidence of bladder cancer (BC) has increased in Japan, and the incidence rate was 20 and 5 per 100,000 of the population for males and females, respectively (1). Approximately $75-85 \%$ of patients with BC are initially diagnosed with non-muscle-invasive disease (NMIBC) (2). Non-muscle-invasive BC has a tendency to recur $(50-70 \%)$ and may progress $(10-20 \%)$ to muscle-invasive disease (MIBC) in time, which is considered to be associated with a poor prognosis (3). In particular, high-grade disease accounts for approximately $10 \%$ of all NMIBC. High-grade NMIBC has a high risk of progression and can lead to cancer death (4). When NMIBC is treated by transurethral resection alone, the recurrence rate is $50-80 \%$, and $20-60 \%$ of patients have a risk of progression to MIBC (5). Therefore, the discrimination of patients with high-grade NMIBC with the potential to progress to MIBC is clinically important for aggressive clinical therapy in the early stage (6). However, there are currently no surrogate prognostic markers for patients with NMIBC patients. In addition, it is difficult to assess the prognosis of patients with high-grade NMIBC by only a biopsy specimen and urinary cytology (7-9). For these reasons, one of the most important clinical challenges is the identification of novel biomarkers for aggressive high-grade NMIBC destined to recur and progress following initial treatment that can be used to predict the outcome of patients.

Tumor-associated antigens released into the bloodstream are able to induce a humoral immune response and generate autoantibodies (10). Interestingly, tumor antigens are undetectable in sera at the early stage of tumorigenesis; the nature of an antibody amplification response to antigens makes tumor-associated autoantibodies promising early 
Table I. Clinicopathological characteristics of patients with bladder cancer.

\begin{tabular}{llcc}
\hline & & \multicolumn{2}{c}{ Number of patients (\%) } \\
\cline { 3 - 4 } & & Serum & Tissue \\
\hline \multirow{2}{*}{ Gender } & Male & $73(77)$ & $93(81)$ \\
& Female & $22(23)$ & $22(19)$ \\
Age, years & Median & 71 & 65 \\
Stage & Range & $29-88$ & $40-82$ \\
& HG-NMIBC & $63(66)$ & $28(24)$ \\
Histological grade & MIBC & $32(34)$ & $87(76)$ \\
& High & $71(75)$ & $51(44)$ \\
Carcinoma in situ & Low & $24(25)$ & $64(56)$ \\
& Negative & $90(95)$ & $101(88)$ \\
Nodal status & Positive & $5(5)$ & $14(12)$ \\
& Negative & $82(86)$ & $83(77)$ \\
Lymphovascular invasion & Positive & $13(14)$ & $26(23)$ \\
& Negative & $90(95)$ & $111(97)$ \\
& Positive & $5(5)$ & $3(3)$ \\
\hline
\end{tabular}

MIBC: Muscle-invasive bladder cancer; HG-NMIBC: high-grade nonmuscle-invasive bladder cancer muscle.

biomarkers in cancer diagnosis (11). Hundreds of tumorassociated antibodies have been identified, and the potential utility of autoantibodies as novel biomarkers for cancer diagnosis has been discussed (12). Furthermore, a recent review based on autoantibody profiling of patients with cancer has suggested that autoantibodies can be used as not only diagnostic but also prognostic biomarkers (13).

In this study, we performed two-dimensional gel electrophoresis (2-DE) combined with immunoblot analysis using patient serum as the first antibody to identify tumorassociated antigenic proteins that elicit a humoral response in sera from patients with $\mathrm{BC}$.

\section{Materials and Methods}

Study population. Ninety-five serum samples from patients with BC who had not received any therapy at Kitasato University Hospital Japan from 2005 to 2012 were collected. According to standard hospital procedures, venous blood samples were collected. And after centrifugation at $1,000 \times g$ for $20 \mathrm{~min}$, separated serum samples were collected and stored at $-80^{\circ} \mathrm{C}$ until use. One hundred and fifteen surgically resected primary $\mathrm{BC}$ tissues at Kitasato University Hospital from 1990 to 2004 were used in this retrospective study. The 2002 TNM and WHO classifications (2) were used for determination of the pathological stages and grades of the tumor. Clinical characteristics of $\mathrm{BC}$ patients whose sera and tissues were used in this study are shown in Table I. Thirty-five serum samples from healthy donors were also collected for patients with $\mathrm{BC}$ and stored at $-80^{\circ} \mathrm{C}$ until use. This study was approved by the Ethics Committee of Kitasato University School of Medicine (approval number: C09-504, B17-010). All patients were informed of the aim of the study and gave consent for the use of their samples.
Cell lines and sample preparation. The human bladder cancer cell lines TCCSUP, T24, 5637, and RT4 were purchased from the American Type Culture Collection (Manassas, VA, USA). These cell lines were grown in RPMI-1640 (SIGMA Aldrich Corp., St. Louis, MO, USA) supplemented with $10 \%$ fetal bovine serum (Biowest, Nuaillè, France), 100 units $/ \mathrm{mL}$ of penicillin, and $100 \mu \mathrm{g} / \mathrm{mL}$ of streptomycin (Life Technologies Corp., Carlsbad, CA, USA) at $37^{\circ} \mathrm{C}$ in $5 \% \mathrm{CO}_{2}$ and $95 \%$ humidified air and harvested when they reached confluency. After being washed with phosphate-buffered saline without bivalent ions and centrifuged, cells were stored at $-80^{\circ} \mathrm{C}$. All cells were solubilized in lysis buffer (7 M urea, $2 \mathrm{M}$ thiourea, 2\% 3[(3-cholamidopropyl) dimethylammonio] propanesulfonic acid, 10 $\mathrm{mM}$ tris(2-carboxyethyl)-phosphine hydrochloride (TCEP), $2.5 \%$ pharmalyte at pH 3-10 (GE Healthcare Bio-Sciences Corp., Piscataway, NJ, USA), and one tablet of complete mini EDTA-free protease inhibitors (Roche Diagnostics, Mannheim, Germany) per 10 $\mathrm{mL}$ of solution) using an ultrasonic homogenizer (VP-050; TAITEC Co., Ltd., Saitama, Japan), and centrifuged at $20,000 \times g$ for $30 \mathrm{~min}$ at $4^{\circ} \mathrm{C}$. Finally, the protein concentration was quantified using Protein Assay Reagent (Bio-Rad Laboratories, Hercules, CA, USA).

Two-dimensional gel electrophoresis. 2-DE was performed according to our previous study (14). The first-dimensional agarose isoelectric focusing gel (75 $\mathrm{mm}$ in length and $2.5 \mathrm{~mm}$ in inner diameter) was made with single pharmalyte at $\mathrm{pH}$ 3-10 (GE Healthcare Bio-Sciences Corp.). Twenty-five micrograms of whole protein extract from four cell lines were equally mixed and applied to the cathodic end of the agarose isoelectric focusing gel, and loaded in stepwise voltages as follows: $100 \mathrm{~V}: 20 \mathrm{~min}, 300 \mathrm{~V}: 15 \mathrm{~min}, 500 \mathrm{~V}: 15 \mathrm{~min}, 700 \mathrm{~V}: 60$ min, and $900 \mathrm{~V}$ : $150 \mathrm{~min}$ at $4^{\circ} \mathrm{C}$. After fixation in $10 \%$ trichloroacetic acid and $5 \%$ sulfosalicylic acid for $3 \mathrm{~min}$ at room temperature $(\mathrm{R} / \mathrm{T})$ with mild shaking, agarose gels were placed in distilled water and washed three times for $15 \mathrm{~min}$ each at $\mathrm{R} / \mathrm{T}$. The agarose gel was immersed in equilibration buffer $[0.06 \mathrm{M}$ Tris- $\mathrm{HCl}(\mathrm{pH} 6.8), 2 \%$ sodium dodecyl sulfate (SDS), $10 \%$ glycerol, $5 \%$ 2-mercaptoethanol, and $0.02 \%$ bromophenol blue (BPB)]. The agarose gel was then placed on the top of the second-dimensional $10 \%$ polyacrylamide gel, and loaded with a constant current at $20 \mathrm{~mA}$ per gel.

Two-dimensional gel electrophoresis immunoblotting. The proteins separated on 2-DE gels were transferred to polyvinylidene difluoride (PVDF) membranes (Millipore Corp.) overnight at $\mathrm{R} / \mathrm{T}$ with a constant voltage at $10 \mathrm{~V}$. After blocking with $0.5 \%$ casein for $60 \mathrm{~min}$, the membranes were reacted with 20 -fold-diluted mixed sera of three patients with high-grade NMIBC regardless of disease progression after initial transurethral resection with $0.05 \%$ casein/Tris-buffered saline (TBS) containing $0.1 \%$ tween 20 for $15 \mathrm{~h}$ at $4^{\circ} \mathrm{C}$. The membranes were washed three times with TBS containing $0.1 \%$ tween 20 (TBST) and reacted with 1,000-times-diluted horseradish peroxidase (HRP)-conjugated rabbit anti-human IgG antibody (Dako, Glostrup, Denmark) with $0.05 \%$ casein/TBST for $30 \mathrm{~min}$ at $\mathrm{R} / \mathrm{T}$. After washing three times for $5 \mathrm{~min}$ each with TBST, immunoreactive on the membrane were visualized with stable 3,3'diaminobenzidine (DAB) solution (Life Technologies Corp.) for 15 $\min$ at $\mathrm{R} / \mathrm{T}$. The visualized images were digitized with a highresolution scanner (GT-9800; SEIKO EPSON Corp., Tokyo, Japan).

Identification of proteins recognized by autoantibodies. The identification of antigenic proteins recognized by autoantibodies was performed according to our previous study (15). In brief, the 
proteins separated on 2-DE gels were stained by coomassie brilliant blue (CBB) (PhastGel Blue R; GE Healthcare Bio-Sciences Corp.) solution, and staining images were digitized with a high-resolution scanner. To match the immunoreactive spots on the membrane with protein spots on the gel, both digitized images were overlayed using Adobe Photoshop software (version 7.0; Adobe Systems Inc., San Jose, CA, USA). The protein spots matched with the immunoreactive spots were manually excised from the gel and destained with $50 \%$ acetonitrile $/ 50 \mathrm{mM} \mathrm{NH} \mathrm{NHCO}_{3}$ until they became colorless. After dehydration, the pieces of gel were then rehydrated in $10 \mu \mathrm{l}$ of trypsin solution containing $10 \mathrm{ng} / \mu \mathrm{l}$ trypsin (Trypsin Gold, Mass Spectrometry Grade; Promega, Madison, WI, USA) for $45 \mathrm{~min}$ at $4^{\circ} \mathrm{C}$ and incubated for $24 \mathrm{~h}$ at $37^{\circ} \mathrm{C}$ with a minimum volume of trypsin solution and $7 \mu \mathrm{l}$ of $25 \mathrm{mM}$ $\mathrm{NH}_{4} \mathrm{HCO}_{3}$. After incubation, the digested tryptic peptide solutions were collected. Finally, they were spotted on a Prespotted AnchorChip 96 Set for Proteomics (Bruker Daltonics, Bremen, Germany) and analyzed with matrix-assisted laser desorption/ ionization tandem time-of-flight-mass spectrometry (MALDITOF/TOF MS) using Autoflex III (Bruker Daltonics) and FlexAnalysis software (version 3.0.96; Bruker Daltonics) according to the manufacturer's instructions. The peptide mass fingerprint (PMF) and MS/MS spectra were processed with FlexAnalysis and BioTools software (version 3.0.183; Bruker Daltonics). The combined spectral data were connected with the MASCOT Server (version 2.3; Matrix Science, London, UK) and database searches were run using the IPI human database (version 3.82; 92,104 sequences; 36,547,220 residues, http://www.ebi.ac.uk/IPI/ Databases.html). Mascot scores of a hit above 62 and $p<0.05$ were set as the thresholds for protein identification.

Dot blot analysis. Based on the results of the above proteomic approaches and database or literature-based information, protein phosphatase 1, catalytic subunit, alpha isoform (PPP1CA) protein was selected. The full-length recombinant proteins corresponding to these proteins were synthesized with Gateway entry clones using an in vitro wheat germ cell-free protein synthesis system (16). The recombinant proteins were solubilized in lysis buffer [62.5 mM Tris$\mathrm{HCl}(\mathrm{pH} \mathrm{6.8)}, 2 \%$ SDS, $10 \%$ glycerol, 5\% 2-mercaptoethanol, $0.001 \% \mathrm{BPB}$, and $1 \mathrm{M}$ phenylmethylsulfonyl fluoride] and spotted onto PVDF membranes using a micro-dot-blot arrayer (Kakengeneqs Co., Ltd., Chiba, Japan). After blocking with TBS with $2 \%$ Tween 20 for $60 \mathrm{~min}$ at $\mathrm{R} / \mathrm{T}$, the membranes were reacted with 400 -fold-diluted serum from individual patients with $\mathrm{BC}$ or healthy controls with $0.05 \%$ casein/TBST for $15 \mathrm{~h}$ at $4^{\circ} \mathrm{C}$. The membranes were washed three times with TBST for 5 min each at $\mathrm{R} / \mathrm{T}$ and reacted with 1,000-times-diluted HRP-conjugated rabbit anti-human IgG (Dako) with $0.05 \%$ casein/TBST for $30 \mathrm{~min}$ at $\mathrm{R} / \mathrm{T}$. After washing with TBST three times for 5 min each, signals were developed using Immobilon Western Chemiluminescent HRP Substrate (Millipore Corp.). The signal intensities were analyzed using DotBlotChip System software (version 4.0; Dynacom Co., Ltd., Chiba, Japan). Each normalized signal was represented by the positive intensity minus the background intensity around the spot.

Sodium dodecyl sulfate-polyacrylamide gel electrophoresis (SDS$P A G E)$ immunoblotting. Four BC cell lines were solubilized in lysis buffer [62.5 mM Tris- $\mathrm{HCl}$ (pH 6.8), 2\% SDS, 10\% glycerol, 5\% 2mercaptoethanol, $0.001 \% \mathrm{BPB}, 1 \mathrm{mM}$ phenylmethanesulfonyl fluoride] with an ultrasonic homogenizer. After centrifugation at
$20,000 \times \mathrm{g}$ for $30 \mathrm{~min}$ at $4^{\circ} \mathrm{C}$, the protein concentration was quantified using Protein Assay Reagent. Then, $10 \mu \mathrm{g}$ of extracted proteins was separated by $10 \%$ polyacrylamide gel with a constant current at $20 \mathrm{~mA}$ per gel. The separated proteins on the gels were transferred to PVDF membranes overnight at $\mathrm{R} / \mathrm{T}$ with a constant voltage at $10 \mathrm{~V}$. After blocking with $0.5 \%$ casein for $60 \mathrm{~min}$, the membranes were reacted with 1,000- fold-diluted antibody to PPP1CA (Abcam, Cambridge, MA, USA) or 20,000- fold-diluted anti- $\beta$-actin antibody (Life Technologies Corp.) with $0.05 \%$ casein/TBST overnight at $4^{\circ} \mathrm{C}$. The membranes were then washed three times for 5 min each with TBST and reacted with 1,000-folddiluted HRP-conjugated anti-mouse (Dako) or anti-rabbit IgG antibody (Dako) with $0.05 \%$ casein/TBST for $30 \mathrm{~min}$ at R/T. After washing three times for $5 \mathrm{~min}$ each with TBST, immunoreactive bands on the membrane were detected with Immobilon Western Chemiluminescent HRP Substrate and captured by the ATTO Cool Saver System (ATTO Corp., Tokyo, Japan).

Immunohistochemical staining. After formalin-fixing and paraffinembedding of four $\mathrm{BC}$ cell lines and $115 \mathrm{BC}$ tissues, they were processed into 3 - $\mu \mathrm{m}$-thick sections, and then used for immunohistochemistry. They were deparaffinized in xylene and rehydrated in a descending ethanolic series, and then treated with $3 \%$ hydrogen peroxide for $20 \mathrm{~min}$. Subsequently, the sections were antigen-retrieved by autoclaving in $0.01 \mathrm{M}$ citrate buffer $(\mathrm{pH}$ $6.0) / 0.1 \%$ Tween 20 for $10 \mathrm{~min}$ at $121^{\circ} \mathrm{C}$. After blocking with $2 \%$ normal swine serum (Dako)/TBS ( $\mathrm{pH} \mathrm{7.4)} \mathrm{at} \mathrm{R/T} \mathrm{for} 10 \mathrm{~min}$, the sections were reacted with 500-fold-diluted anti-PPP1CA (Abcam, Cambridge, MA, USA) with $2 \%$ normal swine serum/TBS overnight at $4^{\circ} \mathrm{C}$. The sections were then washed three times for 5 min each with TBS and reacted with 1,000-fold-diluted biotinylated secondary antibody (LSAB2 kit/HRP; Dako) for $30 \mathrm{~min}$ at R/T. After washing three times for $5 \mathrm{~min}$ each with TBS, the immunoreactivity was visualized by stable DAB solution and counterstained with Mayer's hematoxylin. The nuclear staining of PPP1CA in mitotic tumor cells served as an internal control. By comparison with nuclear staining in the internal control cells, the staining intensity was scored as 0 (negative), 1+ (weaker), 2+ (equivalent), or $3+$ (stronger). In a preliminary study, we assessed the discriminative value for $\mathrm{BC}$ characteristics and prognosis using each score as the cutoff point. Univariate analysis revealed that a cutoff value scored $2+$ or $3+$ for tumor cells accounting for more than $30 \%$ of tumor cells in the tissue provided the best positive and negative prediction for $\mathrm{BC}$ progression and survival (data not shown).

Statistical analysis. Significant differences between clinical characteristics and serum IgG levels of autoantibodies were assessed by the Mann-Whitney $U$-test, and those between clinical characteristics and immunohistochemical staining scores were assessed by the chi-square test. The area under the curve (AUC) and best cut-off point were calculated employing receiver-operating characteristic curve (ROC) analysis. Cumulative survival rates of patients were estimated using the Kaplan-Meier method, and the significance of survival differences was tested using the log-rank test. Multivariable analysis was performed by employing the Cox proportional hazards regression model. A $p$-value of less than 0.05 was used to determine the level of significance. All statistical analyses were performed using StatFlex software version 6.0 (Artech Co., Ltd., Osaka, Japan). 


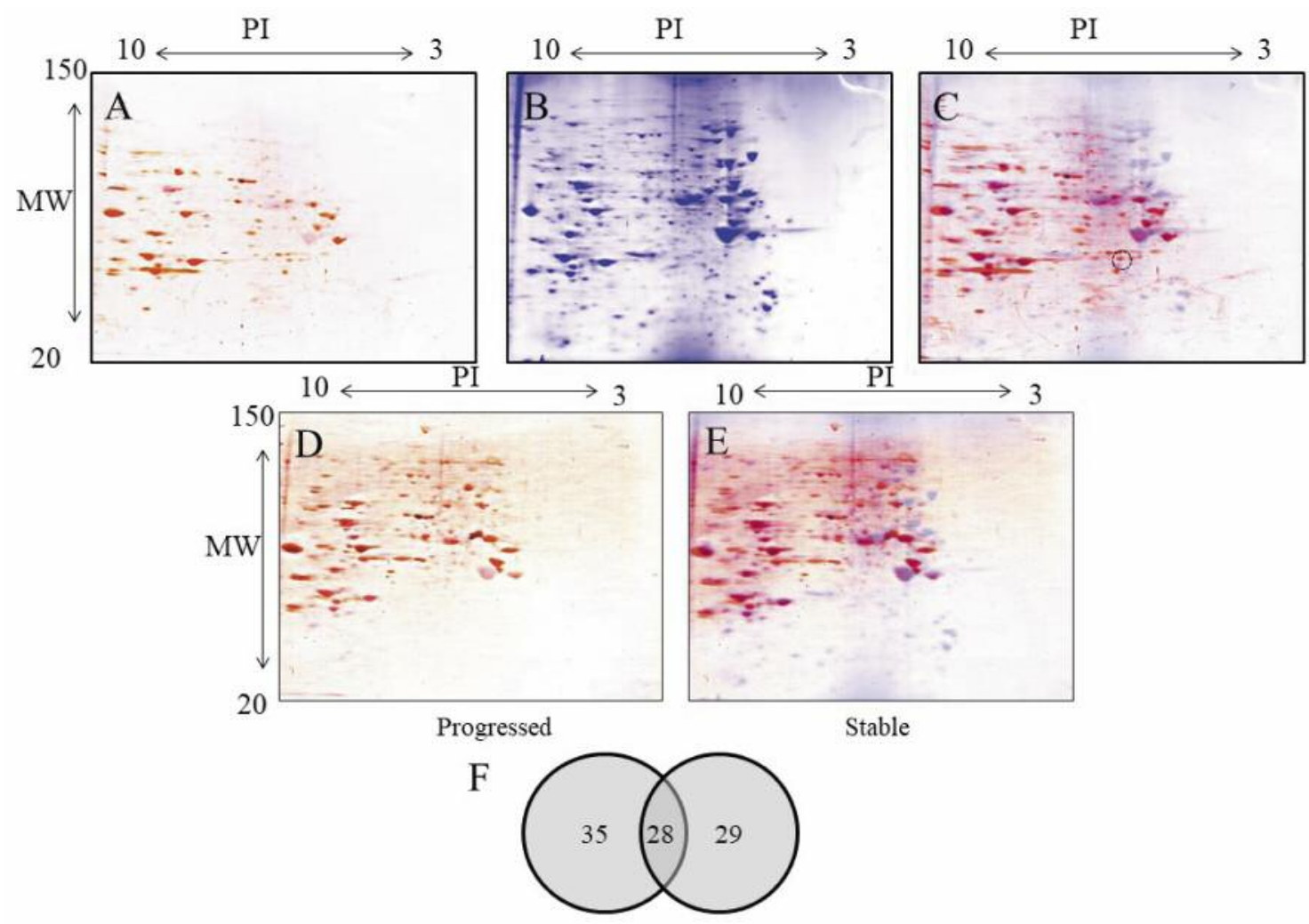

Figure 1. Detection of proteins recognized by autoantibodies in sera from patients with bladder cancer (BC) by Two-dimensional gel electrophoresis (2-DE) immunoblot analysis. The whole protein extract from four BC cell lines were mixed and separated by 2-DE and transferred to polyvinylidene difluoride membranes. The membranes were incubated with mixed pre-treated sera from patients with BC whose disease had progressed (A) or was stable $(C)$. Protein patterns of CBB-stained 2-DE gel (B). Merged images of immunoreactive spots on the membranes and Coomassie Brilliant Blue $(C B B)$-stained 2-DE gels $(C, E)$. By comparing immunoreactive patterns, 35 and 29 spots were specifically detected in sera from patients with highgrade non-muscle-invasive $B C$ whose disease had and had not progressed afterinitial surgical resection, respectively. Twenty-eight proteins were common to both groups $(D, F)$. The protein spots that matched immunoreactive ones on the 2-DE gel were excised and identified by Matrix-Assisted Laser Desorption/Ionization Tandem Time-of-Flight-Mass Spectrometer. PI: Isoelectric Point; MW: Molecular Weight.

\section{Results}

Expression of 2-DE immunoblotting. The whole proteins extracted from the four $\mathrm{BC}$ cell lines were mixed and separated by 2-DE and transferred onto the PVDF membranes. They were reacted with mixed sera of three patients each with HG-NMIBC regardless of disease progression after initial transurethral resection (Figure 1A and D). We detected 92 immunoreactive spots in total, of which 35 were detected only in sera of patients with highgrade NMIBC whose disease had progressed and 29 whose disease had not progressed after initial transurethral resection. Twenty-eight proteins were common to both groups (Figure 1F). The protein spots that matched immunoreactive spots on the membrane were excised from CBB-stained 2-DE gel (Figure 1B-E), and underwent in-gel digestion and MALDI-TOF/TOF MS analysis. As a result,
35 and 27 proteins were identified (Table II) from the immunoreactive spots in patients with and without disease progression, respectively.

Distribution of PPPICA levels using dot-blot analysis. We synthesized PPP1CA recombinant protein that were detected only in sera from patients with high-grade NMIBC whose disease had progressed after initial transurethral resection. Serum IgG levels for each autoantibody in 95 patients with $\mathrm{BC}$ and 35 normal controls were investigated by dot-blot analysis with these recombinant proteins. In univariate analysis, an optimal cut-off value of 49.0 was applied and showed that the serum IgG levels of anti-PPP1CA autoantibodies were significantly higher in patients with $\mathrm{BC}$ than in healthy controls ( $p<0.001$, Figure $2 \mathrm{~A})$. The serum IgG levels of anti-PPP1CA autoantibody were significantly higher in patients with MIBC than in those with NMIBC 
Table II. Antigenic proteins identified in sera of patients with bladder cancer.

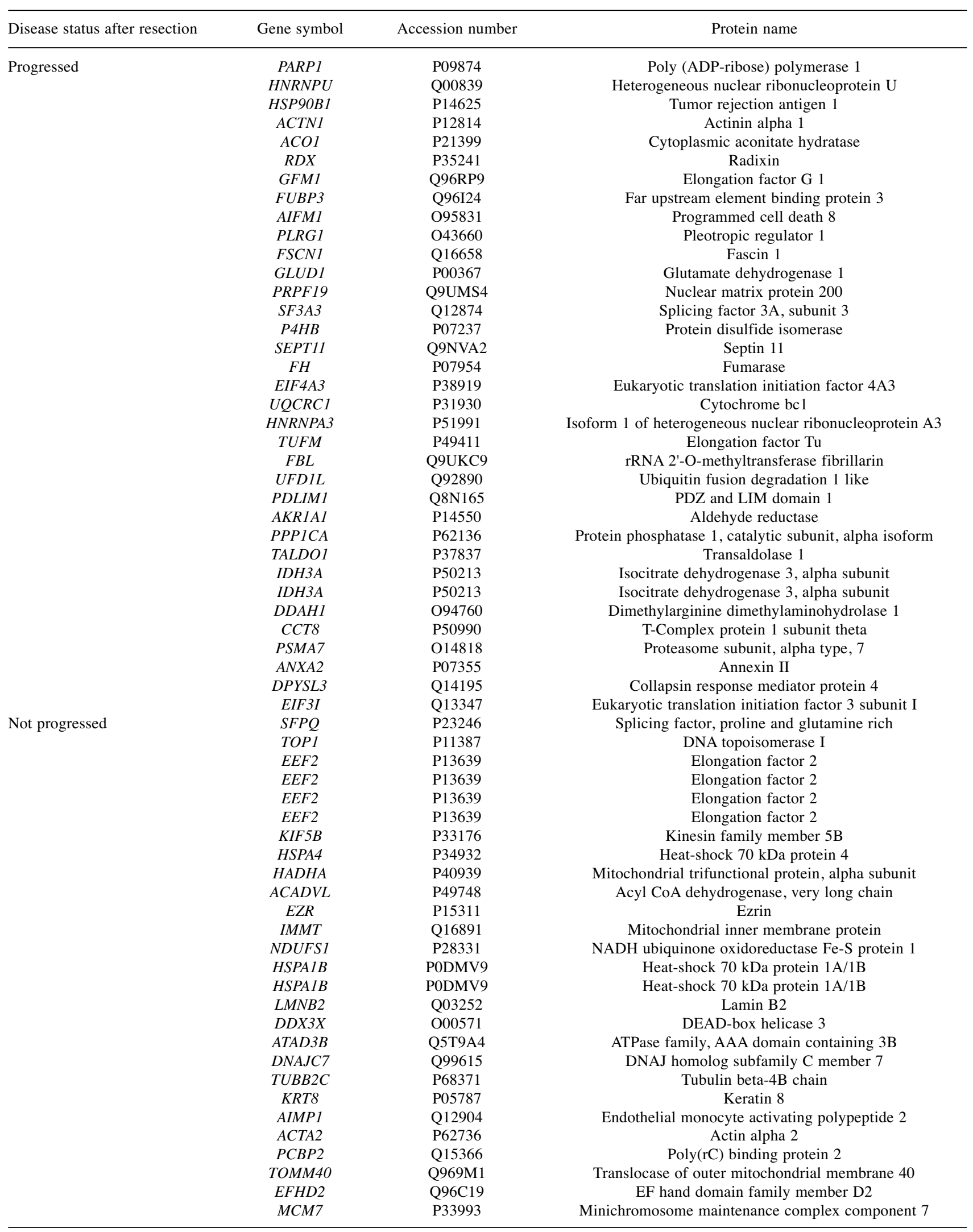



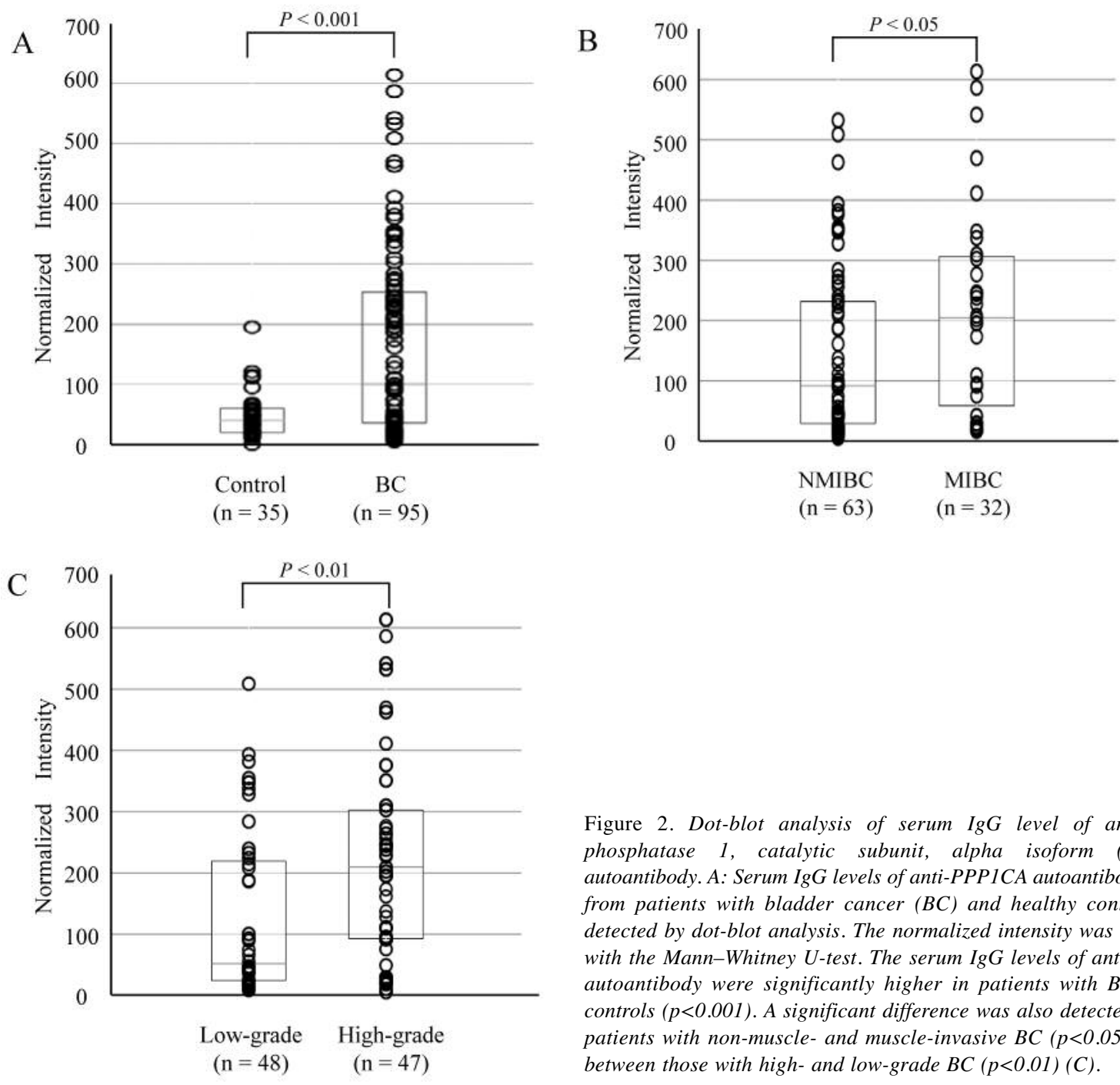

Figure 2. Dot-blot analysis of serum IgG level of anti-protein phosphatase 1, catalytic subunit, alpha isoform (PPP1CA) autoantibody. A: Serum IgG levels of anti-PPPICA autoantibody in sera from patients with bladder cancer $(B C)$ and healthy controls were detected by dot-blot analysis. The normalized intensity was calculated with the Mann-Whitney U-test. The serum IgG levels of anti-PPPICA autoantibody were significantly higher in patients with $B C$ than in controls $(p<0.001)$. A significant difference was also detected between patients with non-muscle- and muscle-invasive $B C(p<0.05)(B)$, and between those with high-and low-grade $B C(p<0.01)(C)$.

$(p<0.05$, Figure 2B), and those with high-grade than in those with low-grade BC $(p<0.01$, Figure $2 \mathrm{C})$. No significant difference between the serum IgG levels of two other autoantibodies of patients with BC patients and controls was detected (data not shown).

Cut-off value using ROC analysis. Based on ROC analysis of anti-PPP1CA autoantibody, an optimal cut-off value of 49.0 was applied. The resulting diagnostic sensitivity and specificity for BC was 64.2 and $65.7 \%$, respectively. The AUC for anti-PPP1CA autoantibody in patients with BC compared to normal controls was 0.72 (95\% confidence interval: 0.676-0.763, Figure 3A).

Association of serum PPPICA levels with prognosis. To estimate whether serum IgG levels of anti-PPP1CA

autoantibody have an independent predictive value for recurrence-free or cancer-specific survival of patients with $\mathrm{BC}$, uni- and multivariable-analyses were performed. At a median follow-up of 62.3 (range=2-166.4) months, KaplanMeier projection indicated that an increase of serum $\mathrm{IgG}$ level of anti-PPP1CA autoantibody was significantly correlated with higher cancer-specific survival and recurrence-free survival ( $p<0.05$, respectively, Figure 3B and C). Multivariate analysis with Cox proportional hazards regression analysis revealed that the serum IgG level of antiPPP1CA autoantibody and pathological stage were significantly correlated with cancer-specific survival $(p<0.05$, respectively, Table III).

The expression of PPPICA in bladder cancer cell lines and tissues. The expression levels of PPP1CA protein in $\mathrm{BC}$ cell 
lines were confirmed by immunoblotting analysis and immunohistochemical staining. Immunoblotting analysis revealed that the expression of PPP1CA protein was higher in high-grade BC cell lines (TCCSUP and T24) than in lowgrade ones (5637 and RT4) (Figure 4A). By immunohistochemical staining, nuclear and cytoplasmic staining of PPP1CA protein, especially strong nuclear staining in mitotic cells, was observed. The staining intensity of nuclear PPP1CA protein was higher in high-grade TCCSUP and T24 cells than in low-grade 5637 and RT4 cells. However, no differences in cytoplasmic staining were observed between the two groups (Figure 4B). The expression of PPP1CA protein in BC tissues was also confirmed by immunohistochemical staining. In normal urothelial cells, weakly positive nuclear and cytoplasmic staining was observed in a scattered pattern (Figure 4Bi and ii). In contrast, nuclear and cytoplasmic staining of PPP1CA protein was observed in most tumor cells at various levels from case to case (Figure 4Biii and iv), and strong staining was noted in mitotic cells (Figure 4Bv).

The association of PPP1CA level with clinocopathological findings. Univariate analysis revealed that nuclear staining of PPP1CA protein was significantly higher in MIBC than in NMIBC $(p<0.01)$, and in high-grade than in low-grade BC $(p<0.001)$ (Table IV). Nuclear staining was also higher in tumors with lymphovascular invasion $(p<0.05)$. However, no significant correlation between PPP1CA expression and prognosis was observed.

\section{Discussion}

Autoantibodies against tumor-associated antigens have been identified in sera from patients with colon, breast, lung, ovary, bladder and several other cancer types (16-21). Thus, the application of the humoral immune response for the detection of cancer biomarkers has important potential and has been suggested as ideal screening for cancer diagnosis with high-level prognostic value $(12,13)$. Furthermore, the immune system is especially well adapted for the early detection of cancer because autoantibodies can be detected before the appearance of other biomarkers or phenotypic alternations at an early stage of tumorigenesis (11). In several types of carcinoma, the associations between the levels of autoantibodies in sera from patients and clinicopathological factors including prognosis have been reported, and the serum levels of autoantibodies were found to be independent prognosis indicators.

In the present study, we performed 2-DE/immunoblot analysis to identify antigenic proteins that are recognized by autoantibodies in sera from patients with high-grade NMIBC regardless of disease progression after initial transurethral resection. We picked up a total of 92 immunoreactive spots

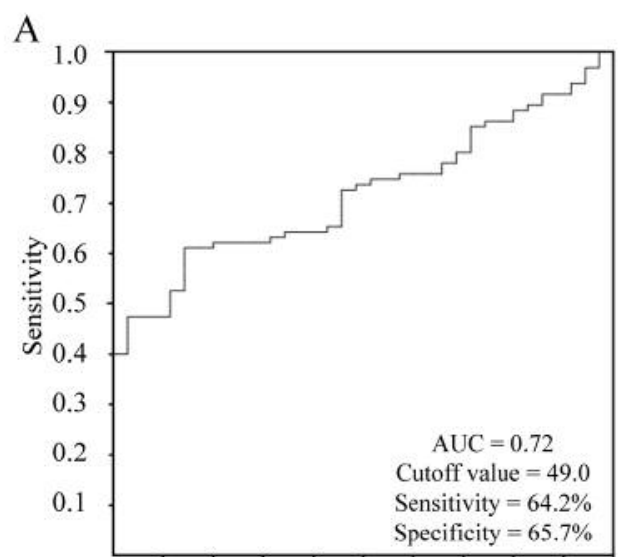

$\begin{array}{lllllllllll}0.1 & 0.2 & 0.3 & 0.4 & 0.5 & 0.6 & 0.7 & 0.8 & 0.9 & 1.0\end{array}$ 1 - Specificity
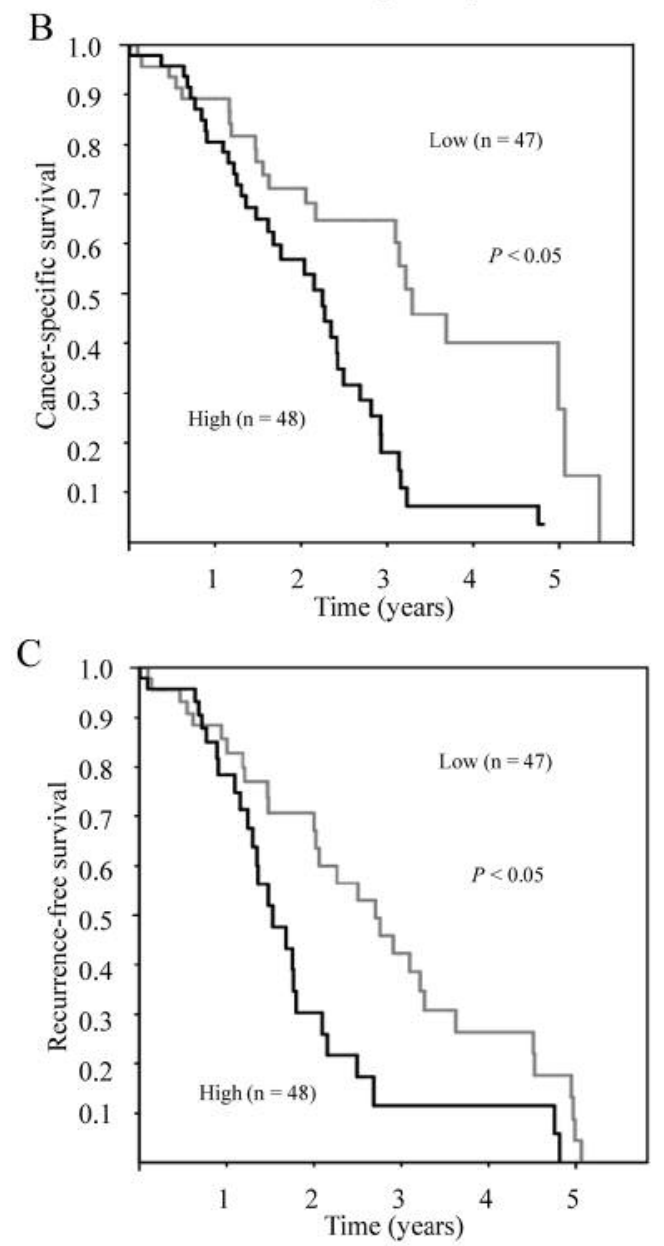

Figure 3. Receiver operating characteristic (ROC) analysis of bladder cancer $(B C)$ and the probability of cancer-specific survival according to serum IgG level of anti protein phosphatase 1, catalytic subunit, alpha isoform (PPP1CA) autoantibody. The corresponding area under the ROC curve of serum IgG level of anti-PPP1CA autoantibody in patients with $B C$ compared to healthy controls was 0.72 (A). Significant correlation was revealed between the serum IgG level of anti-PPP1CA autoantibody and recurrence-free survival (B), as well as cancerspecific survival $(C)$ of patients with $B C$. 
A
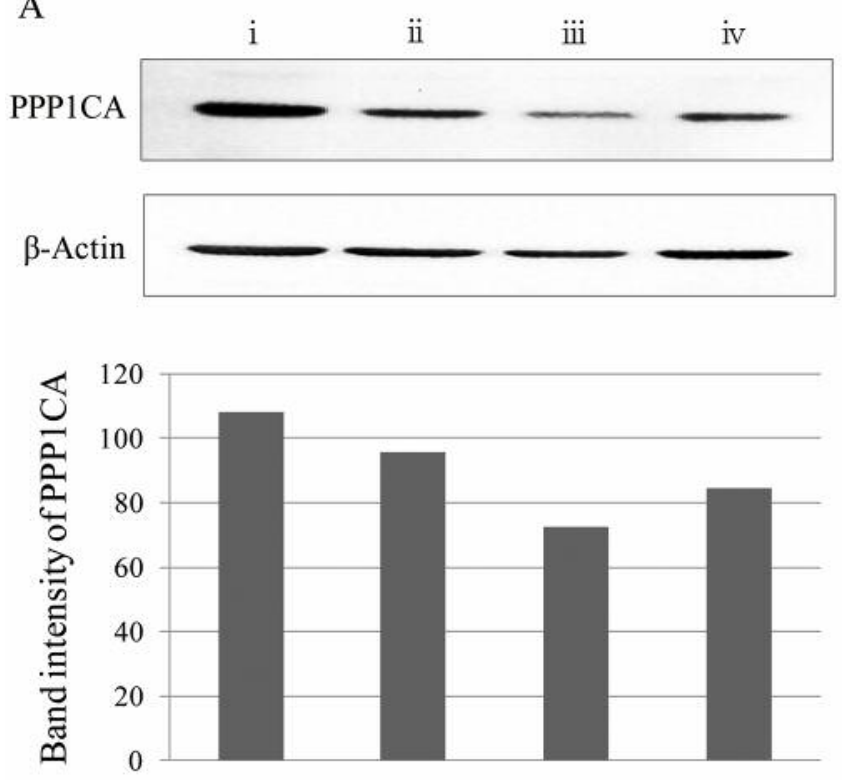

B
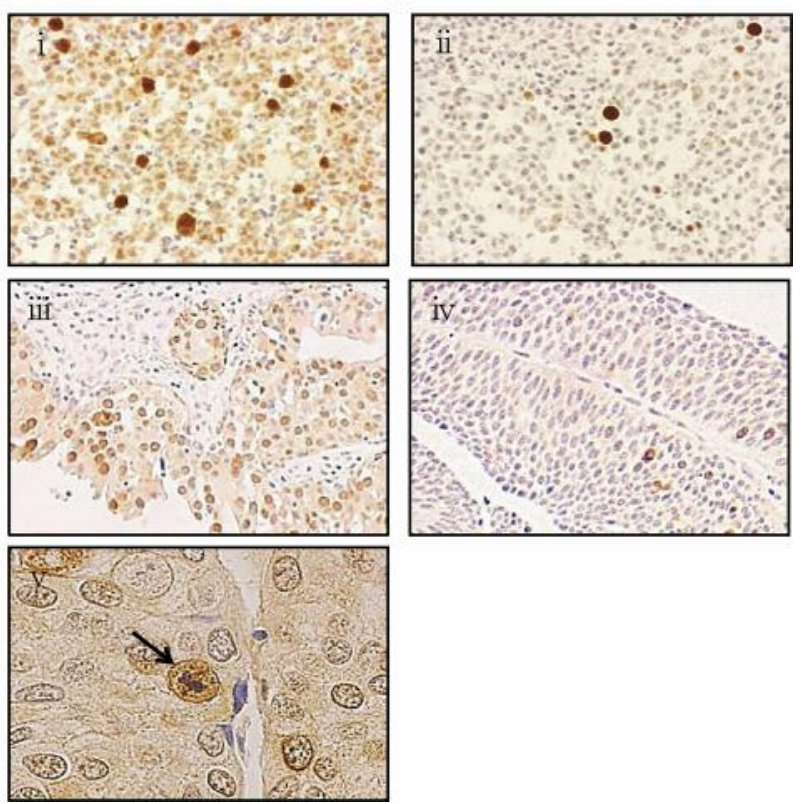

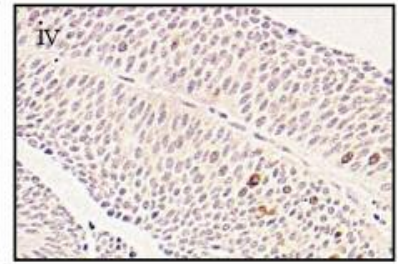

Figure 4. Expression of protein phosphatase 1, catalytic subunit, alpha isoform (PPP1CA) protein in bladder cancer (BC) cell lines (A) and tissues $(B)$. Immunoblotting $(A)$ and immunohistochemical staining $(B)$ were performed to confirm the expression of PPP1CA in BC cell lines and tissues. A: Expression was strong in high-grade TCCSUP (i) and T24 (ii) cells, but much weaker in low-grade 5637 (iii) and RT4 (iv) cells. B: In normal urothelial cells, weakly positive nuclear and cytoplasmic staining was observed in a scattered pattern ( $i$ and ii). In BC tissues, expression of PPP1CA protein was observed in the nucleus and cytoplasm of tumor cells (iii and iv), and the strongest staining was observed in the mitotic cells ( $v$, arrow).

Table III. Multivariate Cox proportional hazards regression analyses for prediction of recurrence and survival in patients with bladder cancer.

\begin{tabular}{|c|c|c|c|c|c|c|c|}
\hline \multirow[b]{2}{*}{ Factor } & \multirow[b]{2}{*}{ Subgroup } & \multicolumn{3}{|c|}{ Recurrence-free survival } & \multicolumn{3}{|c|}{ Cancer-specific survival } \\
\hline & & HR & $95 \% \mathrm{CI}$ & $p$-Value & HR & $95 \% \mathrm{CI}$ & $p$-Value \\
\hline Anti-PPP1CA autoantibody & High $v s$. low & 0.53 & $0.16-1.74$ & 0.12 & 4.06 & $1.19-13.9$ & 0.03 \\
\hline Gender & Male vs. female & 0.91 & $0.43-1.96$ & 0.77 & 0.46 & $0.63-2.78$ & 0.46 \\
\hline Pathological stage & NMIBC $v s$. MIBC & 1.7 & $0.79-3.69$ & 0.14 & 0.02 & $1.21-7.86$ & 0.02 \\
\hline Histological grade & High $v s$ low & 1.84 & $0.67-5.04$ & 0.21 & 0.84 & $0.33-2.46$ & 0.84 \\
\hline Nodal status & Positive $v s$. negative & 0.59 & $0.18-1.93$ & 0.30 & 0.19 & $0.13-1.50$ & 0.19 \\
\hline Lymphovascular invasion & Positive $v s$. negative & 0.72 & $0.13-4.04$ & 0.81 & 0.22 & $0.05-1.96$ & 0.22 \\
\hline
\end{tabular}

HR: Hazard ratio; CI: confidence interval; NMIBC: Non-muscle-invasive bladder cancer muscle; MIBC: muscle-invasive bladder cancer.

that differed between patients with different progression patterns, and revealed that serum IgG levels of anti-PPP1CA autoantibody were significantly higher in patients with BC than in healthy controls. In addition, we also immunohistochemically confirmed the in vivo expression of PPP1CA protein in tumor cell lines and BC tissues.

PPP1CA is one of four major serine/threonine-specific protein phosphatases identified in eukaryotic cells (23), and is involved in signal transduction, apoptosis, protein synthesis, and cell-cycle regulation $(24,25)$. Detailed roles of PPPICA gene expression in tumorigenesis are unclear, but it was reported that overexpression of PPPICA gene enhanced the growth of oral, breast, and ovarian cancer cells $(26,27)$. In contrast, overexpression of PPPICA gene inhibited the growth of renal and colorectal carcinoma cells (28). In glioblastoma, the expression of PPP1CA protein was increased in histologically high-grade astrocytoma compared to normal brain tissue and low-grade astrocytoma (29). In prostate cancer, PPP1CA protein expression was increased in tumor tissues compared with benign 
Table IV. Association of protein phosphatase 1, catalytic subunit, alpha isoform (PPP1CA) expression in tissue with clinicopathological factors.

\begin{tabular}{|c|c|c|c|}
\hline \multirow[b]{2}{*}{ Characteristic } & \multicolumn{2}{|c|}{ PPP1CA } & \multirow[b]{2}{*}{$p$-Value } \\
\hline & $\begin{array}{c}\text { Positive } \\
\text { n (\%) }\end{array}$ & $\begin{array}{c}\text { Negative } \\
\mathrm{n}(\%)\end{array}$ & \\
\hline Total & & $69(60)$ & $46(40)$ \\
\hline \multicolumn{4}{|l|}{ Gender } \\
\hline Male & $54(47)$ & $39(34)$ & \multirow[t]{2}{*}{0.38} \\
\hline Female & $15(13)$ & $7(6)$ & \\
\hline \multicolumn{4}{|l|}{ Age } \\
\hline$\geq 60$ Years & $42(37)$ & $36(31)$ & \multirow[t]{2}{*}{0.051} \\
\hline$<60$ Years & $27(23)$ & $10(9)$ & \\
\hline \multicolumn{4}{|c|}{ Pathological stage } \\
\hline NMIBC & $10(9)$ & $18(16)$ & \multirow[t]{2}{*}{$<0.01$} \\
\hline MIBC & $59(51)$ & $28(24)$ & \\
\hline \multicolumn{4}{|c|}{ Histological grade } \\
\hline High & $50(43)$ & $14(12)$ & \multirow[t]{2}{*}{$<0.001$} \\
\hline Low & $19(17)$ & $32(28)$ & \\
\hline \multicolumn{4}{|c|}{ Carcinoma in situ } \\
\hline Negative & $62(54)$ & $39(34)$ & \multirow[t]{2}{*}{0.42} \\
\hline Positive & $7(6)$ & $7(6)$ & \\
\hline \multicolumn{4}{|l|}{ Nodal status } \\
\hline Negative & $47(41)$ & $36(31)$ & \multirow[t]{2}{*}{0.13} \\
\hline Positive & $19(17)$ & $7(6)$ & \\
\hline \multicolumn{4}{|c|}{ Lymphovascular invasion } \\
\hline Negative & $18(16)$ & $22(19)$ & \multirow[t]{2}{*}{$<0.05$} \\
\hline Positive & $43(37)$ & $19(17)$ & \\
\hline
\end{tabular}

NMIBC: Non-muscle-invasive bladder cancer; MIBC: muscle-invasive bladder cancer.

hyperplastic tissues, and its expression was also associated with a high Gleason score (30). In BC, it has been reported that the expression levels of PPPICA mRNA in urinary sediment were increased when compared with healthy controls, and positive correlation between PPPICA mRNA expression and tumor recurrence was indicated (31). However, to our knowledge, the expression of PPP1CA protein in $\mathrm{BC}$ and its serum autoantibody level have never been reported.

Therefore, we examined the expression of PPP1CA protein in $\mathrm{BC}$ tissue and the serum IgG levels of antiPPP1CA autoantibody. The serum IgG levels of antiPPP1CA autoantibody were associated with less favorable clinicopathological factors of $\mathrm{BC}$, suggesting it to be an independent predictor of poorer survival of patients with $\mathrm{BC}$. Similarly, the expression of PPP1CA protein in BC tissue was also associated with poorer clinicopathological parameters of BC. Therefore, as far as we are aware of, this is the first report regarding the sero-diagnostic and prognostic potential of autoantibody to PPP1CA in BC. Further studies are needed to clarify the more detailed role of PPP1CA in tumorigenesis.
In conclusion, we identified several proteins that were recognized by autoantibodies in the sera of patients with HGNMIBC by proteomic analysis, and found that serum IgG levels of anti-PPP1CA autoantibody were significantly higher in patients with $\mathrm{BC}$ than in healthy controls. In addition, higher serum IgG levels of anti-PPP1CA autoantibody were also associated with muscular invasion, a higher tumor grade, and poorer prognosis of patients with BC. Furthermore, the expression of PPP1CA protein in BC tissue was also correlated with muscle invasion, a higher grade, and lymphovascular invasion by the tumors. These data suggest that serum anti-PPP1CA autoantibody is a candidate sero-diagnostic and prognostic marker in patients with BC. However, further studies are required to reveal the clinical utility of higher serum IgG levels of anti-PPP1CA autoantibody for management of patients with BC.

\section{Acknowledgements}

This study was supported, in part, by JSPS KAKENHI Grant (number 23590414, 24592408 and 15K10607) and the JSTSENTAN (Development of Systems and Technology for Advanced Measurement and Analysis: Life Innovation Area) Program from the Japan Science and Technology Agency, the Japan Society for the Promotion of Science fellows (25-5519), the 2013-2014 Project Study from the Graduate School of Medical Sciences, Kitasato University.

\section{References}

1 Kakehi Y, Hirao Y, Kim WJ, Ozono S, Masumori N, Miyanaga N, Nasu Y and Yokomizo A: Bladder Cancer Working Group report. Jpn J Clin Oncol 40(Suppl 1): i57-64, 2010.

2 Kirkali Z, Chan T, Manoharan M, Algaba F, Busch C, Cheng L, Kiemeney L, Kriegmair M, Montironi R, Murphy WM, Sesterhenn IA, Tachibana $M$ and Weider J: Bladder cancer: epidemiology, staging and grading, and diagnosis. Urology 66: 4-34, 2005.

3 Witjes JA and Hendricksen K: Intravesical pharmacotherapy for non-muscle-invasive bladder cancer: a critical analysis of currently available drugs, treatment schedules, and long-term results. Eur Urol 53: 45-52, 2008.

4 van den Bosch S and Alfred Witjes J: Long-term cancer-specific survival in patients with high-risk, non-muscle-invasive bladder cancer and tumour progression: a systematic review. Eur Urol 60: 493-500, 2011

5 Emiliozzi P, Pansadoro A and Pansadoro V: The optimal management of T1G3 bladder cancer. BJU Int 102: 1265-1273, 2008.

6 Fritsche HM, Burger M, Svatek RS, Jeldres C, Karakiewicz PI, Novara G, Skinner E, Denzinger S, Fradet Y, Isbarn H, Bastian PJ, Volkmer BG, Montorsi F, Kassouf W, Tilki D, Otto W, Capitanio U, Izawa JI, Ficarra V, Lerner S, Sagalowsky AI, Schoenberg M, Kamat A, Dinney CP, Lotan Y and Shariat SF: Characteristics and outcomes of patients with clinical $\mathrm{T} 1$ grade 3 urothelial carcinoma treated with radical cystectomy: results from an international cohort. Eur Urol 57: 300-309, 2010. 
7 Matsumoto K, Ikeda M, Hirayama T, Nishi M, Fujita T, Hattori M, Sato Y, Ohbu M and Iwamura M: Clinical value of dividing false-positive urine cytology findings into three categories: atypical, indeterminate, and suspicious of malignancy. Asian Pac J Cancer Prev 15: 2251-2255, 2014.

8 Tilki D, Burger M, Dalbagni G, Grossman HB, Hakenberg OW, Palou J, Reich O, Roupret M, Shariat SF and Zlotta AR: Urine markers for detection and surveillance of non-muscle-invasive bladder cancer. Eur Urol 60: 484-492, 2011.

9 Volpe A, Racioppi M, D’Agostino D, Cappa E, Gardi M, Totaro A, Pinto F, Sacco E, Marangi F, Palermo G and Bassi PF: Bladder tumor markers: a review of the literature. Int J Biol Markers 23: 249-261, 2008.

10 Fernandez Madrid F: Autoantibodies in breast cancer sera: candidate biomarkers and reporters of tumorigenesis. Cancer Lett 230: 187-198, 2005.

11 Heo CK, Bahk YY and Cho EW: Tumor-associated autoantibodies as diagnostic and prognostic biomarkers. BMB Rep 45: 677-685, 2012.

12 Hanash S: Harnessing immunity for cancer marker discovery. Nat Biotechnol 21: 37-38, 2003.

13 Kobold S, Luetkens T, Cao Y, Bokemeyer C and Atanackovic D: Prognostic and diagnostic value of spontaneous tumor-related antibodies. Clin Dev Immunol 2010: 721531, 2010.

14 Nagashio R, Sato Y, Jiang SX, Ryuge S, Kodera Y, Maeda T and Nakajima T: Detection of tumor-specific autoantibodies in sera of patients with lung cancer. Lung Cancer 62: 364-373, 2008.

15 Minami S, Nagashio R, Ueda J, Matsumoto K, Goshima N, Hattori M, Hachimura K, Iwamura M and Sato Y: Detection of tumorassociated antigens in culture supernatants using autoantibodies in sera from patients with bladder cancer. Biomed Res 35: 25-35, 2014.

16 Goshima N, Kawamura Y, Fukumoto A, Miura A, Honma R, Satoh R, Wakamatsu A, Yamamoto J, Kimura K, Nishikawa T, Andoh T, Iida Y, Ishikawa K, Ito E, Kagawa N, Kaminaga C, Kanehori K, Kawakami B, Kenmochi K, Kimura R, Kobayashi M, Kuroita T, Kuwayama H, Maruyama Y, Matsuo K, Minami K, Mitsubori M, Mori M, Morishita R, Murase A, Nishikawa A, Nishikawa S, Okamoto T, Sakagami N, Sakamoto Y, Sasaki Y, Seki T, Sono S, Sugiyama A, Sumiya T, Takayama T, Takayama Y, Takeda H, Togashi T, Yahata K, Yamada H, Yanagisawa Y, Endo Y, Imamoto F, Kisu Y, Tanaka S, Isogai T, Imai J, Watanabe $\mathrm{S}$ and Nomura $\mathrm{N}$ : Human protein factory for converting the transcriptome into an in vitro-expressed proteome. Nat Methods 5: 1011-1017, 2008.

17 Dai N, Cao XJ, Li MX, Qing Y, Liao L, Lu XF, Zhang SH, Li Z, Yang YX and Wang D: Serum APE1 autoantibodies: a novel potential tumor marker and predictor of chemotherapeutic efficacy in non-small cell lung cancer. PLoS One 8: e58001, 2013.

18 Gumus E, Erdamar S, Demirel G, Horasanli K, Kendirci M and Miroglu C: Association of positive serum anti-p53 antibodies with poor prognosis in bladder cancer patients. Int J Urol 11: 1070-1077, 2004.

19 Lacombe J, Mange A and Solassol J: Use of autoantibodies to detect the onset of breast cancer. J Immunol Res 2014: 574981, 2014.
20 Liu W, Li Z, Xu W, Wang Q and Yang S: Humoral autoimmune response to IGF2 mRNA-binding protein (IMP2/p62) and its tissue-specific expression in colon cancer. Scand J Immunol 77: 255-260, 2013.

21 Song L, Zhang SL, Bai KH, Yang J, Xiong HY, Li X, Liu T and Liu HR: Serum agonistic autoantibodies against type-1 angiotensin II receptor titer in patients with epithelial ovarian cancer: a potential role in tumor cell migration and angiogenesis. J Ovarian Res 6: 22, 2013.

22 Zaenker $\mathrm{P}$ and Ziman MR: Serologic autoantibodies as diagnostic cancer biomarkers - a review. Cancer Epidemiol Biomarkers Prev 22: 2161-2181, 2013.

23 Damer CK, Partridge J, Pearson WR and Haystead TA: Rapid identification of protein phosphatase 1-binding proteins by mixed peptide sequencing and data base searching. Characterization of a novel holoenzymic form of protein phosphatase 1. J Biol Chem 273: 24396-24405, 1998.

24 Janssens V and Goris J: Protein phosphatase 2A: a highly regulated family of serine/threonine phosphatases implicated in cell growth and signalling. Biochem J 353: 417-439, 2001.

25 Klumpp S and Krieglstein J: Serine/threonine protein phosphatases in apoptosis. Curr Opin Pharmacol 2: 458-462, 2002.

26 Hsu LC, Huang X, Seasholtz S, Potter DM and Gollin SM: Gene amplification and overexpression of protein phosphatase 1alpha in oral squamous cell carcinoma cell lines. Oncogene 25: 55175526, 2006.

27 Lu Z, Wan G, Guo H, Zhang X and Lu X: Protein phosphatase 1 inhibits p53 signaling by dephosphorylating and stabilizing Mdmx. Cell Signal 25: 796-804, 2013.

28 Castro ME, Ferrer I, Cascon A, Guijarro MV, Lleonart M, Ramon y Cajal S, Leal JF, Robledo M and Carnero A: PPP1CA contributes to the senescence program induced by oncogenic Ras. Carcinogenesis 29: 491-499, 2008.

29 Ladha J, Donakonda S, Agrawal S, Thota B, Srividya MR, Sridevi S, Arivazhagan A, Thennarasu K, Balasubramaniam A, Chandramouli BA, Hegde AS, Kondaiah P, Somasundaram K, Santosh V and Rao SM: Glioblastoma-specific protein interaction network identifies PP1A and CSK21 as connecting molecules between cell cycle-associated genes. Cancer Res 70: 6437-6447, 2010.

30 Prowatke I, Devens F, Benner A, Grone EF, Mertens D, Grone HJ, Lichter P and Joos S: Expression analysis of imbalanced genes in prostate carcinoma using tissue microarrays. Br J Cancer 96: 82-88, 2007.

31 Brems-Eskildsen AS, Zieger K, Toldbod H, Holcomb C, Higuchi R, Mansilla F, Munksgaard PP, Borre M, Orntoft TF and Dyrskjot L: Prediction and diagnosis of bladder cancer recurrence based on urinary content of hTERT, SENP1, PPP1CA, and MCM5 transcripts. BMC Cancer 10: 646, 2010.

Received September 5, 2017

Revised September 22, 2017

Accepted September 27, 2017 